\title{
Are Collectivistic Cultures More Prone to Rapid Transformation? \\ Computational Models of Cross-Cultural Differences, Social Network Structure, Dynamic Social Influence, and Cultural Change
}

\author{
Michael Muthukrishna ${ }^{1}$ and Mark Schaller ${ }^{2}$ \\ ${ }^{2}$ London School of Economics \\ ${ }^{2}$ University of British Columbia
}

\begin{abstract}
Societies differ in susceptibility to social influence and in the social network structure through which individuals influence each other. What implications might these cultural differences have for changes in cultural norms over time? Using parameters informed by empirical evidence, we computationally modeled these cross-cultural differences to predict two forms of cultural change: consolidation of opinion majorities into stronger majorities, and the spread of initially unpopular beliefs. Results obtained from more than 300,000 computer simulations showed that in populations characterized by greater susceptibility to social influence, there was more rapid consolidation of majority opinion and also more successful spread of initially unpopular beliefs. Initially unpopular beliefs also spread more readily in populations characterized by less denselyconnected social networks. These computational outputs highlight the value of computational modelling methods as a means to specify hypotheses about specific ways in which cross-cultural differences may have long-term consequences for cultural stability and cultural change.
\end{abstract}


Keywords: cultural differences, cultural change, cultural evolution, conformity, minority influence, social influence, social networks, dynamical systems, computational modeling 


\section{Are Collectivistic Cultures More Prone to Rapid Transformation?}

\section{Computational Models of Cross-Cultural Differences, Social Network Structure, Dynamic Social Influence, and Cultural Change}

There are cross-cultural differences in individuals' attitudes, values, and behavioral dispositions. Might these differences render some societies more susceptible—or more resistant - to change? If so, what specific cross-cultural differences might matter, and what might be their specific implications for cultural change? These questions represent an "important future direction in the study of cultural change" (Varnum \& Grossmann, 2017, p. 965).

It is no easy task to address these kinds of questions. Many population-level cultural changes (e.g., the consolidation of public opinion, the spread of innovative ideas) emerge as cumulative consequences of a great many instances of social influence within complex, highlydynamic systems of interpersonal interaction. Emergent effects of this sort are notoriously difficult to predict. One of the hallmarks of complex dynamical systems is that emergent population-level outcomes not only defy intuitive appraisal, they also cannot be reliably predicted on the basis of the linear if-then rules that govern logical analysis (Kameda, Takezawa, \& Hastie, 2003; Latané, 1996; Mason, Conrey, \& Smith, 2007; Vallacher, Read, \& Nowak, 2002). Complicating matters further is the fact that, just as relevant behavioral dispositions (e.g., individuals' susceptibilities to social influence) may vary across societies, these dispositions also vary across individuals within a society. And complicating matters even further is the fact that, given the structures of social networks, individuals also differ in the number of opportunities they have to be influenced by (and to exert influence on) other individuals. In order to 
articulate — and make plausible predictions about—effects of cultural differences on cultural change, one must account for these variables, and their implications.

Computational modeling methods provide a rigorous means of meeting this conceptual challenge. Computational models allow researchers to identify specific ways in which dynamic processes that unfold over time at one level of analysis might produce emergent properties measurable at another level of analysis (Jackson, Rand, Lewis, Norton, \& Gray, 2017; Vallacher et al., 2002). Although previously-published modelling projects have examined cultural changes of various kinds (Boyd \& Richerson, 1985; Henrich, 2001, 2004; Pfau, Kirley, \& Kashima, 2013; Weisbuch, Deffuant, \& Amblard, 2005)—including some recent work that focuses on implications of cultural differences (De, Nau, Pan, \& Gelfand, 2018)—no previous work has attempted to model all of the kinds of variables identified above.

In this article we describe a novel method of doing so. We demonstrate its utility by modeling cultural differences in individuals' susceptibility to social influence as well as cultural differences in the geometric properties of social networks, and then-in a series of computer simulations - modeling the dynamic consequences of interpersonal influence within these different cultural populations. These computational outputs represent rigorously-generated hypotheses regarding cross-cultural differences in the dynamics of cultural change.

\section{Interpersonal Influence Processes as a Source of Cultural Change}

Cultures change for a variety of reasons (Varnum \& Grossmann, 2017). Some changes in population-level norms might simply be aggregate consequences of individuals' responses to changing ecological circumstances (e.g., Grossmann \& Varnum, 2015). Other changes reflect the cumulative consequences of dynamic social influence processes that transpire when individuals interact with each other (Latané, 1996). The role of social influence processes in 
cultural change is evident in research on minority influence (e.g., Moscovici, 1980), word of mouth (e.g., Berger \& Schwartz, 2011), and cultural transmission mechanisms in cultural evolution (e.g., Chudek, Muthukrishna, \& Henrich, 2015; Henrich, 2001; Muthukrishna \& Henrich, 2016; Muthukrishna, Morgan, \& Henrich, 2016). These programs of research illustrate a common set of core processes: People communicate and, as a consequence, they have opportunities to mutually influence each other's opinions, beliefs and behaviors. At a psychological level of analysis, any resulting influence manifests in individuals' changes of opinions, or in the adoption of new beliefs and behavioral tendencies. At a population level of analysis, these individual-level changes may manifest in changes in popular opinion, or in the emergence of cultural norms characterized by new beliefs and behavior tendencies. It is through this dynamic social influence process that fads and fashions wax and wane, that pockets of public opinion propagate across entire populations, and that radical ideas sometimes catch on and sometimes don't (Harton \& Bourgeois, 2003; Kashima, Wilson, Lusher, Pearson, \& Pearson, 2013; Latané, 1996).

At the psychological level of analysis, the outcome of social influence-whether an individual does or doesn't change a particular belief or behavior - may reflect a wide range of distinct psychological processes. Conformity to majority opinion is a potent and common form of social influence; but, sometimes, individuals reject majority opinion and instead adopt opinions expressed by persistent minorities (Nemeth, 1986; Wood, Lundgren, Ouellette, Busceme, \& Blackstone, 1994). Here, when modeling social influence outcomes, we do so in a way that allows the potential for either conformity or minority influence, and which ensures that the former is a substantially more likely outcome than the latter. This operationalization is consistent with empirical evidence showing that minority influence tends to occur only under 
relatively rarified conditions, and reflects the centrality of conformity processes in models of both social influence (Flache et al., 2017) and cultural evolution (Chudek et al., 2015).

We examine the dynamic implications of social influence processes for two specific forms of cultural change: (a) consolidation of existing opinion majorities, and (b) diffusion and spread of new ideas.

\section{Consolidation of Existing Opinion Majorities}

One form of cultural change is the tendency for existing opinion majorities to become even bigger majorities over time — the phenomenon that Latané (1996) labeled consolidation. Consolidation of majority opinion emerges as a consequence of individuals' inclinations to conform to the actions, attitudes, and opinions that they perceive in the majority of others (MacCoun, 2012; Muthukrishna et al., 2016). Individuals who are already in agreement with the perceived majority tend to maintain that opinion over time; individuals whose personal opinions are at variance with the majority feel pressure to change and to adopt the majority opinion instead. Thus, in the absence of countervailing pressures, the size of opinion majorities within a population tends to become incrementally greater over time. This consolidation phenomenon is relevant to many specific outcomes of considerable societal importance (e.g., "bandwagon effects" in electoral politics; (Kenney \& Rice, 1994; Nadeau, Cloutier, \& Guay, 1993).

Although any majority opinion has the potential to consolidate into an even bigger majority (Latané, 1996), this potential may be more readily realized in some societies more than in others. No prior work has directly addressed this possibility, nor identified specific crosscultural differences that might predict the speed of consolidation. 


\section{Diffusion and Spread of New Beliefs}

Another form of cultural change occurs when new ideas, radical beliefs, and novel ways of doing things spread through a population — the phenomenon that sociologists refer to as the diffusion of innovations (Rogers, 2003). Diffusion processes are of substantial relevance to consumer behavior (Berger \& Schwartz, 2011; Brown \& Reingen, 1987), to the success or failure of public health interventions (e.g., Haider \& Kreps, 2004), and to the popular ascendance of novel ideologies and religious beliefs (e.g., Collar, 2007). Not all innovations do spread, of course. The conformity processes that underlie consolidation of majority opinion can pose a psychological barrier to the spread of initially unpopular opinions or beliefs or behavioral practices (Eriksson, Enquist, \& Ghirlanda, 2007). And yet, as research on minority influence reveals, this barrier can be breached (Centola, Becker, Brackbill, \& Baronchelli, 2018; Wood et al., 1994); and some innovations do diffuse throughout entire populations (Rogers, 2003; Wejnert, 2002).

Although scant, some empirical research suggests that there may be cultural differences in the diffusion of new things within a society. Wilken, Miyamoto, and Uchida (2011) found that the popularities of cultural products - books, music, baby names — changed more rapidly in Japan than in the United States. Another study compared the rates at which novel consumer products became popular within different European nations that varied on a measure of individualism/collectivism (Dwyer, Mesak, \& Hsu, 2005). Results showed that, after controlling for economic differences between countries, there was a general tendency for new technologies to catch on more quickly in countries characterized by more highly collectivistic values. An intriguing implication (which, at first glance, may appear inconsistent with the finding that 
collectivism is associated with conformity to existing norms; e.g., Bond \& Smith, 1996) is that cultural change may occur more rapidly within collectivistic cultures.

Although provocative, those few empirical results reflect highly specific cultural changes that occurred in specific cultural contexts. Computational modeling can help to ascertain whether those results might plausibly reflect a more broadly generalizable cultural phenomenon; and, if so, computational models can also help to articulate possible underlying psychological processes.

Some recent computational modelling projects have produced results predicting crosscultural variability in the adoption of new cultural norms (De et al., 2018). This work, which is summarized in the next section - is relevant to our own models, which focus on cultural differences in individuals' susceptibility to social influence and on cultural differences in the social networks through which this influence occurs.

\section{Cultural Differences in Individuals' Susceptibility to Social Influence}

Empirical research shows that, compared to people in individualistic cultures, people in collectivistic cultures are generally more susceptible to the influence of others. Indeed, some questionnaire measures of collectivism include items that explicitly assess the extent to which people seek advice from, and are influenced by, their friends and family members (e.g., Singelis, Triandis, Bhawuk, \& Gelfand, 1995). Additionally, people in more collectivistic cultures are less committed to self-consistency, which contributes to susceptibility to others' influence (HoshinoBrowne, 2012; Spencer-Rodgers, Williams, \& Peng, 2010; Wilken et al., 2011). This cultural difference in influenceability has implications for different kinds of social influence phenomena. Most obviously, people in collectivistic cultures are more likely to conform to majority opinion (Bond \& Smith, 1996). Additionally, under some conditions, people in collectivistic cultures 
may also be more susceptible to minority influence and to other forms of interpersonal persuasion (Ng \& Van Dyne, 2001; Orji, 2016).

These cultural differences in influenceability might plausibly affect the pace of cultural change. Attesting to this possibility are modeling results described by De et al. (2018). Working within a game-theoretic framework, De et al. (2018) modeled cultural differences in individuals' tendency to conform to a majority of others even at the expense of sacrificing personal benefit, and computed consequences for the rate of norm change within a population. These models showed that (compared to populations defined by relatively low levels of conformity) populations defined by higher levels of conformity are likely to exhibit greater initial resistance to norm change and also a greater likelihood of exhibiting a "tipping point" phenomenon in which large numbers of individuals suddenly adopt a new norm.

The models described in De et al. (2018) are innovative and the results are important, but they are not without limitations. Modelling outputs are necessarily contingent upon the specific ways in which conceptual variables and processes are mathematically operationalized, and it is useful to construct additional models that employ different methods to operationalize key variables and processes. In the computer simulations described below, we used novel methods to model cross-cultural differences in individuals' susceptibility to social influence, and to model their implications (for consolidation of majority opinion and for the diffusion of initially unpopular new beliefs).

One notable feature of the models employed by De et al. (2018) is that those models assumed that all individuals were connected to all other individuals, and so all individuals had equal opportunities to influence (and be influenced by) all other individuals with a population. Another feature is that these were homogenous populations where all individuals were equally 
susceptible to influence. While these assumptions had the pragmatic benefit of making those models mathematically tractable, they represent conceptual limitations too. People vary in the extent to which they are influenced by others and, to the extent to which they are influenced, they are influenced primarily by the subset of people who comprise their own personal network of acquaintances (e.g. Cullum \& Harton, 2007; Kashima et al., 2013). In order to rigorously model the effects that cross-cultural differences might plausibly have for cultural change, it is useful to model the structure of social networks.

\section{Cultural Differences in the Structure of Social Networks}

Empirical evidence from many different kinds of populations_-ranging from small-scale aboriginal societies to massive online communities - show that human social networks have several defining structural properties that, collectively, reflect the density of those networks (Apicella, Marlowe, Fowler, \& Christakis, 2012; Henrich \& Broesch, 2011; Ugander, Karrer, Backstrom, \& Marlow, 2011). One property refers to the frequency distribution of the number of acquaintances that individuals have (the "degree distribution"). Because most individuals have at least a few acquaintances, but few individuals have an extremely large number of acquaintances, real social networks are characterized by a degree distribution skewed to the right. A second property refers to the likelihood that any two acquaintances of any individual will also be acquainted with each other. Within real social networks, this likelihood is non-zero, which is reflected in indices that assess the "clustering" of social connections within the network. A third property refers to the average smallest number of social connections required to trace a path from any one individual to any other individual within the population (the "average path length"). For instance, the online social network Facebook - a means of communication and 
social influence in contemporary populations - has an average path length between 3 and 4 (Backstrom, Boldi, Rosa, Ugander, \& Vigna, 2012; Boldi \& Vigna, 2012).

In the computer simulations described below, we attempted to model social network structures such that they were characterized by degree distributions, clustering indices, and average path lengths that might serve as a reasonable proxy for the network structures that underlie human interactions and interpersonal influence. To do so, we employed a novel modelling method that roughly mimicked processes through which social network structures might emerge. The complex geometry of social networks reflects the fact that individuals vary in their trait-like tendencies to form social connections with other individuals. We modelled these individual differences, which then allowed such social network structures to emerge within simulated populations.

This methodology allowed us also to model cross-cultural differences in social network structure. In collectivistic cultures, individuals' social networks are smaller-they have fewer acquaintances_ — and are less densely connected (Choi, Kim, Sung, \& Sohn, 2011; Chua \& Morris, 2006; Liu, Chan, Qiu, Tov, \& Tong, 2018), findings that imply cultural differences in population-level social network structure ${ }^{1}$. Therefore, just as we modeled cultural differences in influenceability, we also modeled cultural differences in social network structure. Doing so allowed us to computationally ascertain whether cultural differences in social network structure might plausibly have independent implications for the speed of cultural change (and also whether cultural differences in social network structure might plausibly moderate any effects produced by cultural differences in influenceability).

\footnotetext{
${ }^{1}$ Cultural differences in social networks may not be attributable to differences in individualism / collectivism, per se, but may instead be attributable to associated differences in personality traits. Collectivism, for example, negatively correlates with extraversion (Hofstede \& McCrae, 2004), a behavioral trait that is positively associated with social network size (Pollet, Roberts, \& Dunbar, 2011).
} 


\section{Additional Conceptual Considerations}

The results of the computational models described below represent rigorously-derived conceptual hypotheses regarding effects that cross-cultural differences (in influenceability and social network structure) might plausibly have on cultural change. Their conceptual utility depends substantially on their robustness. If a specific result occurs only under a very narrow set of circumstances (defined by specific parameters within the computational models) its plausibility and/or range of applicability may be questionable. If the result occurs under a wider range of circumstances, its plausibility and potential range of application is correspondingly higher. Therefore, in addition to modelling the variables described above, we also systematically varied additional parameters in order to test the robustness of the results.

One of these parameters was attentive to the relation between individuals' influenceability and their dispositional tendency to form social connections (which had implications for social network structure). Empirical evidence is equivocal in regard to whether these two dispositional tendencies are independent or possibly negatively correlated (Aluja, García, \& García, 2003; Arpaci, Baloğlu, \& Kesici, 2018). Therefore, we systematically varied whether individuals' tendencies to form social connections were or weren't independent of their susceptibility to social influence.

Second, we were attentive to empirical results showing that, while there is some tendency for people to prefer to affiliate with others who have similar attitudes (Byrne, 1971), the magnitude of this "homophily" effect varies depending on the features of those attitudes (Tesser, 1993) and also varies across cultures (Heine, Foster, \& Spina, 2009; Schug, Yuki, Horikawa, \& Takemura, 2009). Therefore, we systematically varied whether individuals' initial opinions 
were — or weren't—independent of the opinions of others within their personal network of acquaintances, and tested the robustness of results accordingly.

Third, we were attentive to the fact that different models of social influence make different assumptions about decision-rules that govern whether individuals do (or do not) change their opinions in response to information about others' opinions. Some models of social influence imply a roughly linear relationship between the number of people who hold a differing opinion and an individual's likelihood of changing their opinion to match it (for a review, see Flache et al., 2017). Other models (e.g., models of conformist transmission in cultural evolution; Muthukrishna et al., 2016) suggest that people systematically overweight majority opinion and underweight minority opinion, which implies speedier consolidation of majority opinion and stricter barriers against the spread of unpopular beliefs. In order to allow greater opportunity for the spread of unpopular beliefs, we conducted our primary simulations under conditions that modelled a linear relationship. We conducted supplemental simulations under conditions that modeled overweighting of majority opinion.

Finally, when modeling diffusion of initially unpopular beliefs, we were attentive to empirical evidence showing that individuals espousing unpopular beliefs are unlikely to convert others unless they persistently express those unpopular beliefs (Wood et al., 1994). In order to ensure some opportunity for the spread of unpopular beliefs, our primary simulations modeled this unwavering persistence by making one initial adherent entirely non-susceptible to others' influence. We also conducted supplemental simulations under conditions in which this individual's ideological persistence was less absolute. 


\section{Overview of the Computational Modeling Methods}

In order to accomplish the conceptual objectives summarized above, our modeling methods required three distinct phases. In Phase 1 we simulated societies that varied in individuals' susceptibilities to social influence and also in their dispositional tendencies to form new acquaintances. (In doing so, we drew upon empirical evidence in an attempt to model the magnitude of mean differences between cultural populations, as well as individual differences within cultural populations.) Phase 2 modelled the process through which individuals form acquaintances and, as a consequence, social network structure emerges within a population. (Phase 2 therefore also modelled cultural differences in social network structure.) Then, once social network structures were successfully modelled, Phase 3 modelled the process through which individuals influence, and are influenced by, other individuals to whom they are connected within that social network. It was within Phase 3 that we used additional methods to measure the consolidation of majority opinion and the spread of initially unpopular beliefs, and examined how these outcomes may be affected by cultural differences.

In the following sections, we describe these methods, and the emergent consequences. The description of the modelling methods is designed to be accessible to a wide audience, emphasizing the conceptual design of the models with a minimum of mathematical and/or programming detail. (Additional details can be found in Supplemental Materials.) The methods described in the first two sections (Phase 1 and Phase 2) are steps toward the two main parts of our analysis, both of which focus on Phase 3-in which we model the consequences that crosscultural differences (in influenceability and social network structure) have for (a) the consolidation of existing opinion majorities and (b) the spread of initially unpopular new beliefs. 


\section{Phase 1: Modelling Individual Differences and Cross-Cultural Differences}

In our models, we created populations comprised of 900 individuals — a size large enough to be plausibly analogous to meaningful societies, while not so large as to be computationally intractable. Each individual within a simulated population was assigned two numerical values. One value represented the probability that an individual would make a new acquaintance when given the opportunity. This probability value —-which we label "extraversion" for expository ease-was functionally relevant during Phase 2 of our models. ${ }^{2}$ The second value represented the probability that an individual would change a preexisting opinion (or belief or behavioral practice or any other thing that might be responsive to social influence) upon discovering that their acquaintances had a different opinion (or belief, etc.). This probability value-which we label “influenceability" —was functionally relevant during Phase 3 of our models.

In assigning these values, we attempted to accomplish two objectives. (1) Within any single simulation, the distribution of values should plausibly mimic individual differences in behavioral dispositions that exist within any human population; and (2) across different sets of simulations, these distributions should plausibly mimic the magnitude of actual cultural differences in behavioral dispositions, as documented in the empirical literature (e.g., McCrae et al., 2005). To accomplish these objectives, we drew upon the beta distribution (Gupta \& Nadarajah, 2004), which can be used to model both within-population and between-population variability (e.g. Balding \& Nichols, 1995; Batchelder, 1975).

In order to model cross-cultural differences in influenceability (while simultaneously modeling within-culture individual differences in influenceability), we created 3 different beta

\footnotetext{
${ }^{2}$ For the purposes of our models, the broad trait of extraversion is less relevant than the more specific behavioral disposition to form acquaintances. Our decision to label this disposition "extraversion" is informed by empirical evidence that more highly extraverted individuals are more likely to form acquaintances (e.g. Asendorpf \& Wilpers, 1998; Paulhus \& Trapnell, 1998; Selfhout et al., 2010). Other labels might be equally appropriate.
} 
distributions with the following parameter values: $[4,4],[2.5,3.5]$, and $[3.5,2.5]$. The first set of parameters creates a bell-shaped distribution that is symmetrical around a mean value at the midpoint of the probability scale. It represents a "baseline" population. The second set of parameters creates a distribution that is skewed right (i.e., relatively fewer individuals who are highly susceptible to social influence), and has a mean value approximately 0.5 standard deviations less than the baseline population. The third set of parameters creates a distribution that is skewed left (i.e., relatively larger numbers of individuals who are highly susceptible to social influence), and has a mean value approximately 0.5 standard deviations higher than the baseline population. (See Supplemental Materials for more details on these distributions and on beta distributions more generally.)

For each simulation, each of the 900 individuals within the population was randomly assigned an influenceability value drawn randomly from one of these three beta distributions. For some simulations, values were drawn from the $\beta[4,4]$ distribution; these simulations represent cultures with a moderate level of influenceability. For other simulations, values were drawn from the $\beta[2.5,3.5]$ distribution and represent cultures with a relatively low level of influenceability (analogous to highly individualistic cultures). And for still other simulations, values were drawn from the $\beta[3.5,2.5]$ distribution, representing cultures with a relatively high level of influenceability (analogous to highly collectivistic cultures). This procedure simulated individual differences within each simulated population. Also, because differences between the means of the 3 beta distributions mathematically mimic the magnitudes of actual cross-cultural differences in personality traits (McCrae et al., 2005), this procedure was designed to simulate different societies with either moderate, low, or high mean levels of dispositional susceptibility to social influence. (For each of these 3 levels of influenceability, we employed our sampling 
methods to create 10 different 900-individual populations, ensuring that the results of Phase 3 would not be idiosyncratic to any single population of 900 individuals.)

We used an identical procedure to also assign each individual a probability value corresponding to a dispositional tendency to form new social connections ("extraversion"). Within each individual simulation, the procedure simulated individual differences in the tendency to form social connections; and, across different sets of simulations, the magnitude of mean differences mimicked the magnitude of actual cross-cultural differences in this behavioral disposition. Thus - as a consequence of the additional procedures in Phase 2 of our models - we were able to model social network structure and to model cross-cultural differences in social network density.

As a test of robustness, we also systematically varied the extent to which influenceability and extraversion values were correlated within each simulated society. For some simulations, these values were assigned independently. For other simulations, we used an assignment procedure (the copula method for generating multivariate dependence; Kojadinovic \& Yan, 2010) that created a mean within-population correlation of -.3 between influenceability and extraversion values.

\section{Phase 2: Modelling the Emergence of Social Network Structure}

Following the creation of cultural populations, the next phase of our simulations was designed to model social network structure within those populations. There exist many computational algorithms that can lead to the emergence of some kind of network structure (for review see Barabási, 2016; Jackson, 2010), but these algorithms are not typically grounded on principles of human psychology (Schnettler, 2009). In order to accomplish the objectives of this project, we attempted to model the key structural properties of human social networks (skewed 
degree distributions, non-zero levels of clustering and short mean path lengths) in a manner that mimicked the dynamic consequence of individuals' behavioral decisions.

Each simulation began with the 900 individuals located in space on a 30 × 30 grid lattice in the geometric shape of a torus. Each individual was initially assigned exactly four acquaintances: their four closest "neighbors" on the lattice (i.e., the individuals to their immediate east, west, north, and south). We then allowed the model to iterate. On each iteration, each individual $(i)$ had a probability $\left(p_{i}\right)$ —varying between 0 and 1 -of "moving" to an adjacent space on the lattice. If two or more individuals occupied the same space on any iteration, then all individuals occupying the same space at the same time "meet" and would all become "acquaintances". These acquaintances were maintained throughout the rest of the simulation and so, over repeated iterations, individuals had the opportunity to accumulate more and more acquaintances. The formation of acquaintances was computationally constrained in two important ways, both of which are informed by the empirical literature on social interaction:

First, the formation of acquaintances was constrained by proximity. People are more likely to form acquaintances with other people who are closer in geographic space (Festinger, Schachter, \& Back, 1950; Harton \& Bullock, 2007; Latané, Liu, Nowak, Bonevento, \& Zheng, 1995). This "inefficiency" in forming social connections can be modeled in a variety of ways (for examples of geographic network models, see Arentze, Kowald, \& Axhausen, 2013; Butts, Acton, Hipp, \& Nagle, 2012). Our model did so simply by limiting "movement" on the grid geography in any given iteration leading to a higher probability of connecting with those close by. (Note that, given the objectives of Phase 2, the exact computational mechanism itself matters less than geographical constraint itself, and its consequence: Emergence of network structure that serve as a reasonable proxy for the social network structures found in human populations.) 
Second, the probability of forming an acquaintance was constrained by individual differences. Each individual's probability $\left(p_{i}\right)$ of moving to a randomly chosen adjacent space (and thus potentially forming a new acquaintance) was identical to that individual's “extraversion" value (drawn from the beta distribution; see above); these $p_{i}$ values remained constant across iterations. ${ }^{3}$ In sum, the algorithm represents a random walk over a grid lattice where the probability of taking a step in one of four cardinal directions - and thus potentially forming a new acquaintance-is given by an individual's extraversion value.

This model operationalizes two key variables that contribute to the formation of actual social networks; as a result, social network structure emerges as the model iterates. As it iterates further, the social network structure becomes denser. Given the objectives of this phase of the model, we imposed a "stopping rule" before the network structure became too unrealistically dense. Specifically, we stopped Phase 2 of each simulation after 50 iterations. This stopping rule was informed by the results of preliminary exploratory simulations - which revealed that, regardless of the mean level of extraversion within a simulated cultural population, 50 iterations allowed emerging social networks to attain structural properties (degree distribution skew, clustering, path length) that lay within the plausible range of the structural properties that characterize human social networks. See Table 1 for a summary of the properties of social network structures that emerged in populations characterized by low, moderate, and high mean values of extraversion. (See Supplemental Materials for additional details of how network properties change over iterations).

\footnotetext{
${ }^{3}$ It is perhaps worth noting that, in the context of this algorithm, "moving" to an adjacent space does not represent physical mobility per se, but is instead a computational device designed to mimic individuals' likelihood of forming a new acquaintance when provided with the opportunity to do so.
} 
Through these procedures, we modeled social network structures that - as a consequence of our modeling of low, moderate, or high mean levels of extraversion — also mimicked cultural differences in social network structure.

\section{Phase 3: Modelling Interpersonal Influence within Social Networks}

Having created social network structures in Phase 2, these network structures were kept intact throughout the third phase of our models - in which we modeled the cumulative effects of interpersonal influence dynamics on cultural change. Specifically, we modeled the process whereby (a) individuals repeatedly obtain information about the opinions and beliefs of their acquaintances, and potentially (b) update their own opinions and beliefs accordingly (depending upon the extent to which their acquaintances' opinions differ from their own, and depending also upon their own dispositional susceptibility to social influence).

We initiated the third phase of each simulation by assigning one of two possible opinions to each of the 900 individuals within the population. These opinions were binary ( 0 or 1$)$, and so could conceptually represent any opinion, belief, or behavioral tendency that might be subject to social influence. To ensure that our results were not idiosyncratic to the particular initial assignment of opinions, we ran 10 different starting opinion configurations for each of the simulated populations we created. The specific rules for assigning opinions to individuals differed across different sets of simulations. We provide additional details on these assignment rules in the sections below.

We then allowed the model to iterate using a Gillespie algorithm: On each iteration a single individual was randomly selected to be a target of social influence; it required 900 iterations for each individual to have, on average, one opportunity to be the target of influence. (Therefore, for the sake of expository ease, we may consider every set of 900 iterations to 
represent one opportunity for influence.) Being the target of influence meant two things: The individual sampled the opinions of their acquaintances (acquired during Phase 2) in order to obtain information about the distribution of opinions within this set of acquaintances; and the individual then had a probability — varying between 0 and 1 - of changing their existing opinion. This probability was modeled as a joint product of (a) the percentage of acquaintances who held the opposing opinion, and (b) individual's dispositional susceptibility to social influence.

In our primary sets of simulations, the probability $\left(P_{j}\right)$ of an individual $(i)$ acquiring the opposing opinion $j$ was operationalized by the following function:

$$
P_{j}=c_{i} \cdot \frac{b_{j}}{b_{i}+b_{j}}
$$

Where $b_{j}$ is the number of acquaintances who hold the opposite opinion to individual $i, b_{i}$ is the number of acquaintances who hold the same opinion, and $c_{i}$ represents individuals' "influenceability" value (drawn from the beta distribution, with a value lying within a range from 0 to 1 ). The $b_{j}$ and $b_{i}$ values could change from iteration to iteration (depending on the social influence outcomes of previous iterations; the $c_{i}$ values - representing dispositional susceptibility to social influence-remained constant across iterations. ${ }^{4}$

As a robustness check — and as a means of testing the bounds of predictions generated by these simulations — we also conducted supplementary sets of simulations that modeled a tendency for people to systematically overweight majority opinion and underweight minority opinion. In these supplemental simulations, the probability $\left(P_{j}\right)$ of an individual $(i)$ acquiring the

\footnotetext{
${ }^{4}$ It may be helpful to illustrate this formula with an example. Imagine two individuals with influenceability values of .1 and .9 , respectively. If, on one iteration, $90 \%$ of their acquaintances hold the opposing opinion, their likelihoods of subsequently adopting that opposing opinion (a conformity effect) are .09 and .81, respectively. If only $10 \%$ of their acquaintances hold an opposing opinion, their likelihoods of subsequently adopting that opposing opinion (a minority influence effect) are .01 and .09 respectively. Thus, the formula operationalizes opportunities for both conformity and for minority influence, while also operationalizing a much higher likelihood of a conformity outcome (relative to a minority influence outcome).
} 
opposing opinion $j$ was operationalized by the following function (in which the number of acquaintances who held the same and opposite opinions was raised to a power of 1.5):

$$
P_{j}=c_{i} \cdot \frac{b_{j}^{1.5}}{b_{i}^{1.5}+b_{j}^{1.5}}
$$

Using these methods, we operationalized the effects that individuals' dispositional susceptibilities to social influence had on the outcomes of social influence processes. Mean levels of these dispositional susceptibilities varied across different simulated societies. In the following two sections, we describe in detail the implications that these simulated cultural differences had on two distinct kinds of cultural change: Consolidation of majority opinion, and the spread of initially unpopular new beliefs.

\section{Effects of Cultural Differences on Consolidation of Majority Opinion}

\section{Primary Simulations}

What implications might cultural differences in influenceability and social network structure have for the consolidation of majority opinion over time? To address this question, we modelled the process through which, as a result of repeated acts of interpersonal influence, initial small opinion majorities become larger majorities over time. We ran a total of 3600 primary simulations, within which we orthogonally varied population-level mean values of influenceability (low, moderate, and high levels of influenceability; see above), and populationlevel mean values of extraversion (low, moderate, and high levels of extraversion—which, in Phase 2, had consequences for creating population-level differences in social network structure; see above). Thus, 400 simulations each were conducted on 9 simulated cultural populations that varied systematically in terms of influenceability and social network structure.

For the sake of robustness, within each subset of 400 simulations, half of the simulations were run under conditions in which individuals' influenceability and extraversion values were 
assigned independently; and the other half were run under conditions in which these values were negatively correlated $(r=-.3)$ within each simulated population.

We also systematically employed 2 different methods for assigning initial opinions to individuals within each population. One method minimized initial clustering of opinions: Opinions (coded as either 0 or 1) were randomly assigned to each of the 900 individuals within the population. (Because assignment was random, it was very rare that each opinion was held by exactly $50 \%$ of individuals.) The other assignment method was designed to create initial clustering of opinions (the kind of clustering that occurs when individuals preferentially affiliate with others who share their opinions). To do so, we initialized a procedure in which an opinion (coded as 0 or 1) was randomly assigned to a randomly selected individual and also their acquaintances. This procedure iterated until each opinion was held by $50 \%$ of the population (or approximately $50 \%$ when there was no initial clustering).

After initial assignment of opinions, we initialized the simulation of social influence processes (modeled according to Formula 1; see above). As the model iterated—and individuals had the opportunity to be influenced by their acquaintances — one of two opinions eventually endured as the majority, and became an increasingly larger majority. The key question is whether the speed of this consolidation phenomenon differed within different simulated cultural populations (operationalized as differences in influenceability and extraversion in Phase 1).

One means of addressing this question is to identify a threshold for the size of a "supermajority," to measure how many opportunities for influence transpired before a super-majority of that size emerged, and to examine the effects that cultural differences have on that measure. We conducted analyses for a variety of different super-majority thresholds (e.g., 75\%, 90\%), and 
the results were similar regardless of which specific threshold is chosen. We report here the results for a $2 / 3$ super-majority..$^{5}$

We conducted multiple regression analyses to test the main effects of influenceability and extraversion (3 levels each: low, moderate, high), as well as their interaction effect, on the number of influence opportunities required for the $2 / 3$ supermajority to emerge. ${ }^{6}$ Four such analyses were conducted, on 4 subsets of simulations: (a) 900 simulations in which individuals' influenceability and extraversion values were uncorrelated and initial opinions were assigned randomly; (b) 900 simulations in which individuals' influenceability and extraversion values were uncorrelated and initial opinions were clustered; (c) 900 simulations in which individuals' influenceability and extraversion values were negatively correlated and initial opinions were assigned randomly; and (d) 900 simulations in which individuals' influenceability and extraversion values were negatively correlated and initial opinions were clustered.

The results revealed a main effect of influenceability in all 4 subsets of simulations; the magnitude of this effect was similar across all subsets ( $b$ 's ranged from -.26 to -.32; $p$ 's $<.001$ ). In none of these subsets of simulations was there any meaningful main effect of extraversion ( $b$ 's ranged from -.03 to $.03, p$ 's $>.50$ ); furthermore, there weren't any meaningful influenceability $\mathrm{x}$ extraversion interactions ( $b$ 's ranged from -.03 to .06, $p$ 's $>.19$ ). (See Supplemental Materials for additional statistical details.)

\footnotetext{
${ }^{5}$ The $2 / 3$ super-majority corresponds to a decision rule that is commonly used in many real-world decisionmaking contexts. E.g., in the world's two most populous democracies (India and the United States), constitutional amendments require a $2 / 3$ super-majority vote within the relevant voting bodies.

${ }^{6}$ Regression analyses on these results were conducted on log-transformed then scaled values of the outcome variable (number of influence opportunities required for a $2 / 3$ supermajority to emerge), to correct for positive skew. Each simulation is effectively an independent set of circumstances, but to control for any potential nonindependence, we also ran a multilevel model with random intercepts for each constructed network (recall that we ran 10 iterations on each network) with no meaningful change to the results.
} 
The top half of Table 2 summarizes (across all subsets of simulations) the mean number of influence opportunities required for the $2 / 3$ super-majority to emerge in low, moderate, and high-influenceability cultures in these primary simulations. These means show that consolidation of majority opinion occurred more rapidly in cultures characterized by higher mean levels of susceptibility to social influence.

\section{Supplemental Simulations}

The results described above emerged from simulations in which interpersonal influence was modeled by Formula 1 (see above). We conducted a supplemental set of 3600 simulations that were identical in all respects except that interpersonal influence was instead modeled by Formula 2 (which represents a psychological decision-rule that overweights majority opinion and thus leads to faster consolidation of majority opinion) and conducted an analogous set of multiple regression analyses on the outputs.

Results revealed a consistent main effect of influenceability ( $b$ 's ranged from -.25 to -.28; $p$ 's $<.001$ ), and no influenceability x extraversion interactions ( $b$ 's ranged from .03 to $.06, p$ 's $>$ .21). The bottom half of Table 2 summarizes (across all subsets of simulations) the mean number of influence opportunities required for the $2 / 3$ super-majority to emerge in low, moderate, and high-influenceability cultures in these supplemental simulations. These results further attest to the robustness of the finding whereby consolidation of majority opinion occurs more rapidly within cultures characterized by high mean levels of influenceability.

These supplemental simulations also revealed main effects of extraversion-under some circumstances. Specifically, in the subsets of simulations in which individuals' attitudes were assigned randomly, consolidation occurred more rapidly in cultures characterized by lower levels of extraversion $(b$ 's $=.19$ and $.22, p$ 's $<.001)$. No such extraversion effect occurred in the 
subsets of simulations in which individuals' attitudes were initially clustered ( $b$ 's $=-.05$ and .01 , p’s >.21). (See Supplemental Materials for additional statistical details.)

\section{Summary}

These simulations modelled one specific form of cultural change- the consolidation of opinion majorities into "super-majorities." The primary finding was this: Small opinion majorities (starting at close to $50 \%$ ) consolidate more rapidly into super-majorities (held by $2 / 3$ of the population) within cultures characterized by higher mean levels of susceptibility to social influence. Additional simulations would be required to see if starting with a larger majority (e.g., greater than 2/3) would lead to different consolidation behavior, but this effect was highly robust across all conditions simulated here, and emerged regardless of variation in other parameters that were systematically varied across different sets of simulations. (A subset of supplemental simulations produced results showing that, under some very specific circumstances, consolidation also occurred more rapidly in simulated populations defined by lower levels of extraversion. In contrast to the robust effects of cultural differences in influenceability, the extraversion effect was not robust.)

Although the consolidation phenomenon is defined by some incremental change in popular opinion, it also represents a form of cultural stability — a deepening entrenchment of whatever attitudes or ideas were already popular in the first place. A different kind of cultural change occurs when initially unpopular attitudes or beliefs become increasingly popular over time (i.e., the "diffusion of innovations"). In the following section, we report results of computer simulations that model plausible effects of cultural differences for this more radical form of cultural change. 


\section{Effects of Cultural Differences on the Spread of Initially Unpopular New Beliefs}

Despite their numerical disadvantage, some initially unpopular opinions and beliefs do successfully spread within human populations - especially when initial adherents have unshakeable faith in those beliefs and have the means to influence others (Moscovici, 1980; Wood et al., 1994). What implications might cultural differences in influenceability and social network structure have for this diffusion phenomenon? To address this question, we ran more than 300,000 simulations that (employing most of the same methods described above) modelled cultural differences, social network structure, and the outcomes of repeated opportunities for interpersonal influence. But (in contrast to the simulations that focused on consolidation of majority opinion), in these simulations we assigned initial beliefs in such a way that one belief was assigned to the vast majority of individuals within each cultural population, while a different belief was initially highly unpopular. There are many ways in which one might plausibly operationalize this starting condition. We did so as follows: In the primary simulations (all of which employed Formula 1 to operationalize interpersonal influence), this unpopular belief was held by either just a single "lone ideologue" — an initial adherent with unshakeable commitment to that belief — or by that ideologue and a small number of acquaintances. Additional supplementary simulations tested the robustness of findings under conditions in which that initial adherent's commitment to their unpopular belief was not completely unshakeable.

\section{Simulations Involving a "Lone Ideologue"}

A set of 18,000 simulations modeled the diffusion of an unpopular belief held initially by a single individual espousing an unpopular belief (and doing so persistently over time). To do so, in each simulation, we assigned one belief to 899 individuals and a different belief to just 1 individual. In a set of pilot simulations, we confirmed that if this 1 lone adherent was chosen 
randomly, the likelihood of spreading that initially unpopular belief was virtually zero.

Therefore, in order to allow some possibility that the initially unpopular opinion might spread (a necessary precondition for testing effects of cultural differences on this diffusion phenomenon), we assigned the unpopular belief to the individual within each population who had the highest extraversion value (drawn from the relevant beta distribution; see above). We then re-assigned this individual an influenceability value of 0 . By taking these two steps, we ensured that this individual had the means to potentially exert influence on others (because this individual had acquired a large network of acquaintances in Phase 2 of the model), and was resistant to pressure to conform to the countervailing belief initially held by everyone else. ${ }^{7}$

Within this set of 18,000 simulations, we orthogonally varied population-level mean values of influenceability (low, moderate, and high levels of influenceability), and populationlevel mean values of extraversion (low, moderate, and high levels of extraversion—which, in Phase 2, had consequences for creating population-level differences in social network structure). Thus, 2000 simulations each were conducted on 9 simulated cultural populations that varied systematically in terms of influenceability and social network structure. (To ensure robustness of results, for each of these 9 cultural populations, we created 100 separate populations within which we simulated the spread of an initially unpopular belief 10 times each. Additionally, half of all simulations were run under conditions in which individuals' influenceability and extraversion values were assigned independently, and half were run under conditions in which those values were moderately negatively correlated; see above.)

${ }^{7}$ Additional simulations (not reported here) revealed that if interpersonal influence was operationalized by Formula 2-which systematically overweights majority opinion and underweights minority opinion - there was also a vanishingly small likelihood of spreading the initially unpopular belief. Therefore all simulations of the diffusion phenomenon - including the supplemental simulations described below-used Formula 1 to operationalize interpersonal influence. 
After initial assignment of opinions to individuals, we initialized the simulation of social influence processes. As the model iterated - and individuals had the opportunity to be influenced by their acquaintances - there was considerable variability across simulations in the extent to which the initially unpopular belief spread from the lone ideologue to others within the population. The key question here is whether this diffusion phenomenon differed depending on cross-cultural differences in influenceability and social network structure (the latter of which was a function of differences in extraversion).

One means of addressing this question is to identify a specific threshold that defines "successful" diffusion, to measure the percentage of simulations that eventually reached that threshold, and to examine the effects that simulated cultural differences had on that measure. In taking this approach, we defined successful diffusion as $50 \%$ penetration - the point at which an unpopular belief is transformed into a popular one.

We conducted binary logistic regression analyses to test the main effects of influenceability and extraversion (3 levels each: low, moderate, high), as well as their interaction effect, on the percentage of simulations in which the initially unpopular belief successfully spread. Two such analyses were conducted, on 2 subsets of simulations: (a) 9000 simulations in which individuals' influenceability and extraversion values were uncorrelated; and (b) 9000 simulations in which individuals' influenceability and extraversion values were negatively correlated. In both subsets of simulations, results revealed main effects of influenceability (odds ratios $=1.82$ and $1.69, b$ 's $=.60$ and $.53 ; p$ 's $<.001)$, main effects of extraversion (odds ratios $=$ .83 and $.72, b$ 's $=-.19$ and $-.32, p$ 's $<.001)$, and no meaningful interaction effects $(b=-.04$ and $=.01, p ’ s>.45) .($ See Supplemental Materials for additional statistical details.) The nature of these main effects are depicted in Figure 1 (and, more fully, in Table 3): Beliefs that were 
initially held by just a single "lone ideologue" were more likely to spread within cultures characterized by higher levels of influenceability and lower levels of extraversion (i.e., less dense social network structure).

\section{Simulations Involving an Ideologue Accompanied by "Disciples"}

We conducted an additional 216,000 simulations that modelled diffusion of initially unpopular beliefs within contexts in which the ideologue - the unshakeable standard-bearer of an initially unpopular belief — was not initially the only adherent to this unpopular belief, but was instead accompanied by a small band of "disciples". We did so as follows: After first assigning the unpopular belief to the individual with the highest extraversion value (and also re-assigning this individual an influenceability value of 0 ; see above), we then assigned the same belief to either $1,2,3,4,5,6,7,8,9,10,11$, or 12 randomly chosen acquaintances of that individual. (These disciples' influenceability values were unchanged from the values initially drawn from the relevant beta distribution in Phase 1.) All the remaining individuals were assigned the opposite belief. We ran 18,000 simulations for each of the 12 conditions defined by specific numbers of disciples. Within each of these 12 sets of 18,000 simulations, additional populationlevel variables (mean level of influenceability, mean level of extraversion, and whether individuals' influenceability and extraversion values were correlated or not) were systematically varied just as they were within the "lone ideologue" simulations described above. We defined successful diffusion just as we did in the "lone ideologue" simulations, and-for each set of 18,000 simulations - conducted the same statistical analyses on the results.

Results (which are reported in detail in Supplemental Materials) revealed that, although the overall likelihood of successful diffusion increased as the number of initial "disciples" increased, the effects of cultural differences in influenceability and extraversion (i.e., network 
structure) replicated those observed in the "lone ideologue" simulations. Regardless of whether within-population individual differences in influenceability and extraversion were independent or not, an initially unpopular belief was more likely to successfully spread in cultures characterized by higher levels of influenceability (odds ratios ranged from 1.36 to $1.97, p$ 's $<$ .001 ) and lower levels of extraversion (odds ratios ranged from .68 to $.79, p$ 's $<.001$ ). There was no evidence of any consistent interaction.

\section{Supplemental Simulations Involving a Less Ideologically Committed Adherent}

In the primary simulations, described above, we ensured that one adherent of the initially unpopular belief was entirely resistant to social influence (in order to ensure that this "ideologue" persistently maintained that unpopular belief - a typical precondition for successful minority influence; Wood et al., 1994). We ran 72,000 supplemental simulations (within which we systematically varied whether individuals' influenceability and extraversion values were independent or not) to test the robustness of the key results under conditions in which that adherent was not completely resistant to influence.

In one subset of 36,000 simulations, initial beliefs were assigned just as they were in the "lone ideologue" simulations (described above), except that the sole highly-connected adherent of the initially unpopular belief was assigned a non-zero influenceability value. In 18,000 of these simulations, the sole initial adherent was assigned an influenceability value was .01; in another 18,000 simulations, that assigned value was .10. As one might expect, the overall probability of successful diffusion was reduced when initial adherent's influenceability value was .01 (rather than 0) but, as Table 4 reveals, the pattern of effects associated with crosscultural differences in influenceability and extraversion are consistent with those reported above: There were main effects of cross-cultural differences in influenceability (odds ratio $=1.80, b=$ 
$.59, \mathrm{SE}=.05 ; p<.001)$ and extraversion (odds ratio $=.85, b=-.16, p<.001$ ), and no meaningful interaction $(b=.03, p=.56)$. The overall probability of successful diffusion was lower stillapproaching zero-when the initial adherent was assigned an influenceability value was .10 (see Table 4), and there was not enough variability to conduct statistical tests on the effects of influenceability and extraversion (the model reported an overfitting error; singular fit in glmer in lmer R package).

In another subset of 36,000 simulations initial beliefs were assigned just as they were in the simulations involving an "ideologue" accompanied by 12 "disciples" (described above), except that the initial adherent of the initially unpopular belief was assigned an influenceability value was either .01 or .10 (in 18,000 simulations each). Again, when that adherent's influenceability value was .01, there were main effects of both influenceability (odds ratio $=$ $1.31, b=.27, \mathrm{SE}=.03 ; p<.001)$ and extraversion (odds ratio $=.92, b=-.09, p=.001)$, and no meaningful interaction $(b=.04, p=.21)$. And, again, when that adherent's influenceability value was .10 , the overall probability of successful diffusion was much lower and there was not enough variability to conduct meaningful statistical tests.

\section{Summary}

Results revealed that the diffusion of initially unpopular beliefs more readily occurred in cultural populations characterized by higher mean levels of influenceability and also lower mean levels of extraversion (which manifested in fewer mean numbers of acquaintances and less densely-connected social network structures). The latter effect occurs because people are more likely to be influenced by an acquaintance who persistently espouses an unpopular belief if they have a relatively smaller number of other acquaintances (who are likely to hold more conventional beliefs). The former effect reflects the tendency for people who are committed to 
their pre-existing cognitions to resist social influence of any kind, and for people who are less committed to their existing cognitions to be susceptible to any form of social influence. Just as individuals' susceptibility to social influence makes them more susceptible to conformity pressure, it also makes them potentially more susceptible to the influence of an acquaintances who espouse unpopular beliefs. And so, just as the consolidation of majority opinion may occur more rapidly within cultural populations that are more highly influenceable, so too initially unpopular beliefs may be more likely to spread — and to actually become popular —in cultural populations that are more highly influenceable.

In some respects, the latter effect is complementary to a result obtained from models described by De et al. (2018) who found that cultural differences in susceptibility to others' influence — which they modeled in an operationally different way_-predicted a tendency for populations to reach a "tipping point" at which large numbers of people adopt a new norm in a short period of time. Our models differ from those of De et al. (2018) in the operational details and therefore offer a useful complementarity. Not only might more highly influenceable populations be more prone to tipping points, they may also be more prone to the acquisition of new normative beliefs. That big-picture conceptual consistency - along with the consistency of our own results across different sets of simulations that systematically varied other parametersattests to the robustness of this modeling result.

The two main effects produced by our models suggest that (as long as there is some nonzero likelihood that initial adherents can convince others to adopt their initially unpopular belief) novel beliefs and other cultural innovations are likely to spread most rapidly in cultures defined jointly by (a) relatively high susceptibility to social influence and (b) relatively small networks of acquaintances. Empirical evidence indicates that both these features are more characteristic of 
collectivistic cultures than individualistic cultures (e.g., Bond \& Smith, 1996; Choi et al., 2011;

Chua \& Morris, 2006; Liu et al., 2018; Ng \& Van Dyne, 2001; Wilken et al., 2011). The intriguing implication is that, even though collectivistic values are associated with conformity behavior (Bond \& Smith, 1996), collectivistic cultures may nonetheless be more prone to radical cultural change.

\section{General Discussion}

These computer simulations employed novel methods for modeling (a) cross-cultural differences and (b) the consequences that these differences might have on social influence within the context of human social networks, in order to predict (c) their further consequences for cultural change. The primary results—which were robust across a variety of simulated circumstances — revealed that majority opinion consolidated more quickly within cultures characterized by greater susceptibility to social influence, and also that initially unpopular beliefs spread more readily within cultures characterized both by greater susceptibility to social influence and by less dense social network structure (e.g., relatively smaller networks of friends and acquaintances).

The two kinds of cultural change examined here are superficially very different, and so it may seem odd that both forms of cultural change might occur more readily under the same cultural circumstances (high mean levels of susceptibility to social influence). As our models make clear, however, these two kinds of cultural change are responsive to the same underlying psychological considerations. Individuals' susceptibility to social influence predicts their likelihood of conforming to majority opinion and also (more rarely) their likelihood of being persuaded by people who hold unpopular opinions. Consequently, the mean level of "influenceability" with a within a population functions like a kind of lubricant, speeding the pace 
of cultural change - including not just incremental change (of the sort represented by consolidation of majority opinion), but also more truly transformative change (of the sort represented by the widespread diffusion of initially unpopular beliefs).

In conjunction with the modeling results reported by De et al. (2018), the results of our models suggest that — because of variability in mean levels of susceptibility to social influence and also in underlying social network structure-individualistic and collectivistic cultures may be disposed toward different patterns of cultural change over time. Cultural change may occur more slowly and incrementally within individualistic cultures (which are characterized by low levels of influenceability and high social network density). By contrast, in collectivistic cultures (characterized by high levels of influenceability and low social network density), majorities may more rapidly coalesce into monolithic super-majorities; but when this existing orthodoxy is punctured by the spread of heterodox beliefs, this change may proceed at a pace that more closely fits the subjective perception of a "revolution".

\section{Empirical Testability of these Hypotheses}

The results of computer models are not empirical observations. The results summarized above are more aptly characterized as rigorously-derived hypotheses about the effects that cultural differences may have on consolidation of majority opinion and on diffusion of innovations. And, to the extent that these modeling results (or aren't) robust across varying parameters, they provide rigorously-derived insights about whether a hypothesized effect is (or isn't) likely to generalize across a wide range of conditions.

One of these hypotheses - the hypothesis that innovations diffuse more successfully within more collectivistic cultures - is supported by empirical evidence showing that popularities of cultural products changed more rapidly in Japan than in the United States (Wilken et al., 2011) 
and that a country-level index of individual/collectivism predicted the different rates at which novel consumer products became popular within different European countries (Dwyer et al., 2005). Considered in isolation, those empirical results—along with other results documenting other country-level differences in the diffusion of innovations (e.g., von Rosenstiel, Heuermann, \& Hüsig, 2015) — might simply be viewed simply as idiosyncratic bits of evidence documenting highly-specific country-level differences in highly-specific examples of cultural change. But, when considered in the context of the modeling results reported here and by others, including De et al. (2018), those empirical findings appear to be not so idiosyncratic after all. They may represent superficially different manifestations of a cultural change phenomenon that, according to the results of these complementary models, is predicted to occur quite broadly.

It will be useful for future research to test these hypotheses on additional evidence too. These hypotheses are not easy to test, given that they pertain to population-level phenomena that must be documented across long stretches of time. But—as recent empirical research on cultural change reveals (Varnum \& Grossmann, 2017) — these sorts of hypotheses are testable.

\section{Lacunae, Limitations, and Directions for Future Research}

By necessity, conceptual models — including computational models of the sort described here-must omit many of the countless variables that potentially influence individuals' thoughts, feelings, and behavioral decisions. This is not necessarily a limitation (Nowak, 2004). Still, it may be useful to draw attention to specific ways in which our models represent simplified versions of reality, and to consider the implications.

In operationalizing the manner in which individuals assess others' opinions, our models assumed that all acquaintances' beliefs are treated equally. This is not always the case in reality; the pool of opinions that really matter may be smaller than the full set of acquaintances that 
people have. It is reassuring to observe, therefore, that some of the key results observed herethe effects the population-wide mean level of influenceability had on consolidation and diffusion - emerged regardless of the mean level of extraversion, indicating that these effects are robust regardless of the actual number of sources of social influence. Relatedly, when operationalizing the outcomes of social influence (in Formulas 1 and 2), we did not attempt to model variables that may affect whether someone is or isn't a strong source of social influence (e.g., prestige, status, expertise) or whether there might be particular costs or benefits that accrue from adopting the beliefs or behaviors of others (cf. Chudek, Muthukrishna, and Henrich, 2015; Muthukrishna \& Henrich, 2016). Our primary results were robust across a variety of simulated circumstances; in order to determine whether these effects might be moderated by additional variables that might also affect interpersonal influence outcomes, it will be necessary to explicitly incorporate those additional variables within the context of additional models.

Our simulation of social influence processes also assumed that individuals actually obtain information about others' beliefs. In the real world, this is not always the case. For a variety of reasons, some beliefs are more likely than others to be the subject of conversations and other forms of interpersonal communication, and these differences in "communicability" have implications for long-term stability and change in the popularity of these beliefs (Conway \& Schaller, 2007; Schaller, Conway, \& Tanchuk, 2002). The effects obtained from our simulations pertain primarily to attitudes and beliefs that are communicable in some meaningful way. To the extent that beliefs are less communicable, these effects would be expected to be less apparent.

For the primary set of simulations that focused on the diffusion of an initially unpopular belief, we computationally ensured that the primary adherent of that belief was highly committed to maintaining that belief (in order to computationally mimic preconditions for successful 
minority influence). When that ideological commitment was reduced (in supplemental sets of simulations), the likelihood of diffusion was substantially reduced, and the observed effects of cross-cultural differences were reduced accordingly. Analogously, we ensured that this initial adherent had a large number of acquaintances. Had we not done so, the likelihood of successful diffusion — and the effects of cross-cultural differences on successful diffusion—would also have been reduced accordingly. When interpreting these effects on the spread of a radical new belief, it is important to keep in mind the fact that these effects are specific to conditions in which that radical new belief has some reasonable chances of spreading at all.

Note too that our models operationalized social influence in a way that corresponds primarily to the psychological phenomena of conformity and, to a lesser extent, minority influence. Other forms of social influence may have independent effects on changes in cultural norms. For instance, the stability and change in some behavioral norms may be primarily the products of pragmatic constraints pertaining to needs for interpersonal coordination (De, Nau, \& Gelfand, 2017), which are conceptually distinct from the social influence processes that we modeled. Additionally, for psychological reasons that are distinct from those we modeled, individuals are sometimes motivated to deviate from perceived norms, with implications for dynamic changes in popular opinion (e.g., identity signaling; Berger \& Heath, 2007; Berger \& Heath, 2008); and individuals' beliefs also change in response to persuasive messages of various kinds (Albarracín \& Vargas, 2010). To the extent that there are cross-cultural differences bearing on these additional psychological processes and their implications for cultural change, they represent phenomena that are conceptually independent of those examined by our models, and would need to be simulated separately in future models. 
Finally, by modeling cross-cultural differences in susceptibility to social influence and in dispositional tendencies to forge acquaintances, our models barely scratched the surface of the many dispositional differences that might plausibly have implications for social influence processes. For instance, the influential impact of persuasive communications may be moderated by individual differences in needs for cognition and for cognitive closure (e.g., Cacioppo, Petty, Feinstein, \& Jarvis, 1996; Kruglanski, Webster, \& Klem, 1993). Not only do individuals vary in the extent to which they chronically experience these epistemic needs, there are cultural differences too (e.g. Chiu, Morris, Hong, \& Menon, 2000). What implications might these individual and cultural differences have on the cumulative population-level consequences of interpersonal persuasion? In order to sensibly speculate, it will be necessary to develop new models that, while conceptually distinct from our models, might incorporate analogous methodological innovations.

\section{Broader Applications of These Modeling Methods}

As the preceding paragraphs illustrate, the modeling methods that we used are flexible, and can be amended to identify additional hypotheses about possible effects of cultural differences on cultural change. Our modeling methods may have a broader set of useful applications as well.

For example, the methods we used to simulate the emergence of social network structures (in Phase 2 of our simulations) might be profitably amended to model the effects that other variables have on emergent social network structures, and to examine the consequences. Cultural populations are typically comprised of people defined by different demographic categories (gender, ethnicity, language, etc.); these differences affect the formation of relationships that, in turn, affect a wide range of outcomes of considerable psychological and 
societal importance - including prejudice and the acculturation of immigrants (Laar, Levin, Sinclair, \& Sidanius, 2005). The processes can be formalized with the modeling methods that we employed, allowing for rigorous exploration of emergent population-level consequences of demographically constrained patterns of friendship formation (cf. Pfau et al., 2013). More research is required to understand the full effects of these processes on social network structure and more validation analyses are required to understand how well these social network structures match the social network structures found in real human social networks.

These modeling methods might also have useful applications in the study of group decision-making. Although we have applied these methods to research questions bearing on large cultural populations, the methods can be easily amended to address research questions pertaining to smaller groups (Hastie \& Kameda, 2005; Kerr \& Tindale, 2004). For example, recent research shows that the effect of group size on the quality of group decisions depends on the extent to which group members make independent intellectual contributions to these decisions (Kao \& Couzin, 2014). The independence of individuals' contributions is itself likely to depend, in part, on the group's social network structure-which, as we have shown, is influenced by the dispositional traits of group members. With minor amendments, our modeling methods might profitably be used as a means of identifying hypotheses about the effects that individual differences, and cultural differences, may have on group decision-making.

These methods may also have useful applications within the multi-disciplinary study of cultural evolution. Although there are many sophisticated models of cultural evolution (e.g., Boyd \& Richerson, 1985; Henrich, 2004), it is rare for these models to explicitly simulate the geometric properties that define the social network structures of real human populations. For example, recent research reveals relationships between individual-level sociality and emergent 
cultural complexity (Derex \& Boyd, 2016; Kempe \& Mesoudi, 2014; Muthukrishna, Shulman, Vasilescu, \& Henrich, 2013); however, these results were based on models that-like most cultural evolutionary models_-made simplifying assumptions about social network structure governing the interpersonal transmission of cultural information. By incorporating the methods employed in Phase 2 of our models, it may be possible to ask, and answer, questions about the effects of social network structure on cultural transmission and cultural evolution.

\section{Envoi}

Since Homo habilis first banged two rocks together to make a chopping tool, specialized tools have allowed us to overcome the limitations of our bodies. (Hammers let you hit harder; trains let you travel further.) In modern societies, many tools are instrumental in overcoming the limitations of our mental faculties. (Computers let you calculate faster). Most hypotheses in the psychological sciences are generated without such specialized tools, because the typical objects of inquiry (unidirectional causal relations operating at a single level of analysis) are arguably amenable to informal logical deduction. Things are different when addressing questions about phenomena defined by more complex causal relations that play out dynamically over time and produce emergent consequences that must be measured at a different level of analysis entirely. Specialized tools are needed. Computational models provide those tools.

There is a substantial body of computational modeling research identifying populationlevel consequences — representing specific kinds of cultural change — that emerge dynamically from repeated acts of interpersonal influence (e.g., Axelrod, 1997; Mason et al., 2007; Nowak, Szamrej, \& Latané, 1990; Smaldino, 2017; Valente, 1995); but, aside from recent modeling projects by De et al. (2018) no prior research within this tradition had addressed questions about cultural differences on these emergent cultural consequences. There is another substantial body 
of empirical research documenting effects of culture on social influence phenomena (e.g., Bond \& Smith, 1996; Kim \& Markus, 1999; Zou et al., 2009); but that research has focused almost exclusively on short-term individual-level outcomes, without considering further implications for cultural stability and cultural change. Our work—like that of De et al., (2018)—represents a conceptual bridge between these two scholarly literatures. In doing so, it makes novel conceptual contributions to the psychological study of cultural differences and their implications for cultural change. More broadly, it contributes both methodologically and conceptually to multi-disciplinary inquiry into the complex dynamic processes through which ideas spread, norms change, and cultures evolve. 


\section{References}

Albarracín, D., \& Vargas, P. (2010). Attitudes and persuasion: From biology to social responses to persuasive intent. In S. T. Fiske, D. T. Gilbert, \& G. Lindzey (Eds.), The handbook of social psychology (pp. 394-427). Hoboken, NJ: Wiley.

Aluja, A., García, Ó., \& García, L. s. F. (2003). Relationships among extraversion, openness to experience, and sensation seeking. Personality and Individual Differences, 35(3), 671680.

Apicella, C. L., Marlowe, F. W., Fowler, J. H., \& Christakis, N. A. (2012). Social networks and cooperation in hunter-gatherers. Nature, 481(7382), 497-U109.

Arentze, T. A., Kowald, M., \& Axhausen, K. W. (2013). An agent-based random-utilitymaximization model to generate social networks with transitivity in geographic space. Social Networks, 35(3), 451-459.

Arpaci, I., Baloğlu, M., \& Kesici, Ş. (2018). The relationship among individual differences in individualism-collectivism, extraversion, and self-presentation. Personality and Individual Differences, 121, 89-92.

Axelrod, R. M. (1997). The complexity of cooperation: Agent-based models of competition and collaboration. Princeton, NJ: Princeton University Press.

Backstrom, L., Boldi, P., Rosa, M., Ugander, J., \& Vigna, S. (2012). Four degrees of separation. Paper presented at the Proceedings of the 4th Annual ACM Web Science Conference.

Balding, D. J., \& Nichols, R. A. (1995). A method for quantifying differentiation between populations at multi-allelic loci and its implications for investigating identity and paternity. In B. S. Weir (Ed.), Human Identification: The Use of DNA Markers (Vol. 4, pp. 3-12). Dordrecht, Netherlands: Springer Netherlands. 
Barabási, A.-L. (2016). Network science: Cambridge University Press.

Batchelder, W. H. (1975). Individual differences and the all-or-none vs incremental learning controversy. Journal of Mathematical Psychology, 12(1), 53-74.

Berger, J., \& Heath, C. (2007). Where consumers diverge from others: Identity signaling and product domains. Journal of Consumer Research, 34(2), 121-134.

Berger, J., \& Heath, C. (2008). Who drives divergence? Identity signaling, outgroup dissimilarity, and the abandonment of cultural tastes. Journal of Personality and Social Psychology, 95(3), 593-607.

Berger, J., \& Schwartz, E. M. (2011). What drives immediate and ongoing word of mouth? Journal of Marketing Research, 48(5), 869-880.

Boldi, P., \& Vigna, S. (2012, 26-29 Aug. 2012). Four Degrees of Separation, Really. Paper presented at the 2012 IEEE/ACM International Conference on Advances in Social Networks Analysis and Mining.

Bond, R., \& Smith, P. B. (1996). Culture and conformity: A meta-analysis of studies using Asch's (1952b, 1956) line judgment task. Psychological Bulletin, 119(1), 111-137.

Boyd, R., \& Richerson, P. J. (1985). Culture and the evolutionary process. Chicago, IL: University of Chicago Press.

Brown, J. J., \& Reingen, P. H. (1987). Social ties and word-of-mouth referral behavior. Journal of Consumer Research, 14(3), 350-362.

Butts, C. T., Acton, R. M., Hipp, J. R., \& Nagle, N. N. (2012). Geographical variability and network structure. Social Networks, 34(1), 82-100.

Byrne, D. (1971). The attraction paradigm. San Diego, CA: Academic Press. 
Cacioppo, J. T., Petty, R. E., Feinstein, J. A., \& Jarvis, W. B. G. (1996). Dispositional differences in cognitive motivation: The life and times of individuals varying in need for cognition. Psychological Bulletin, 119(2), 197-253.

Centola, D., Becker, J., Brackbill, D., \& Baronchelli, A. (2018). Experimental evidence for tipping points in social convention. Science, 360(6393), 1116-1119.

Chiu, C.-y., Morris, M. W., Hong, Y.-y., \& Menon, T. (2000). Motivated cultural cognition: The impact of implicit cultural theories on dispositional attribution varies as a function of need for closure. Journal of Personality and Social Psychology, 78(2), 247-259.

Choi, S. M., Kim, Y., Sung, Y., \& Sohn, D. (2011). Bridging or Bonding? A cross-cultural study of social relationships in social networking sites. Information, Communication \& Society, 14(1), 107-129.

Chua, R. Y.-J., \& Morris, M. W. (2006). Dynamics of trust in guanxi networks. In R.-Y. Chen (Ed.), National culture and groups (Vol. 9, pp. 95-113). Oxford, United Kingdom: JAI Press.

Chudek, M., Muthukrishna, M., \& Henrich, J. (2015). Cultural Evolution. In D. M. Buss (Ed.), The Handbook of Evolutionary Psychology (2nd ed., Vol. 2). Hoboken, NJ: John Wiley and Sons.

Collar, A. (2007). Network theory and religious innovation. Mediterranean Historical Review, 22(1), 149-162.

Conway, L. G., III, \& Schaller, M. (2007). How communication shapes culture. In K. Fiedler (Ed.), Social communication (pp. 107-127). New York, NY: Psychology Press. 
Cullum, J., \& Harton, H. C. (2007). Cultural evolution: Interpersonal influence, issue importance, and the development of shared attitudes in college residence halls. Personality and Social Psychology Bulletin, 33(10), 1327-1339.

De, S., Nau, D. S., \& Gelfand, M. J. (2017). Understanding norm change: An evolutionary game-theoretic approach. Paper presented at the Proceedings of the 16th Conference on Autonomous Agents and MultiAgent Systems.

De, S., Nau, D. S., Pan, X., \& Gelfand, M. J. (2018). Tipping Points for Norm Change in Human Cultures. arXiv preprint arXiv:1804.07406.

Derex, M., \& Boyd, R. (2016). Partial connectivity increases cultural accumulation within groups. Proceedings of the National Academy of Sciences, 113(11), 2982-2987.

Dwyer, S., Mesak, H., \& Hsu, M. (2005). An Exploratory Examination of the Influence of National Culture on Cross-National Product Diffusion. Journal of International Marketing, 13(2), 1-27.

Eriksson, K., Enquist, M., \& Ghirlanda, S. (2007). Critical points in current theory of conformist social learning. Journal of Evolutionary Psychology, 5(1), 67-87.

Festinger, L., Schachter, S., \& Back, K. (1950). The spatial ecology of group formation. In L. Festinger, K. W. Back, \& S. Schachter (Eds.), Social pressures in informal groups (pp. 33-60). Stanford, CA: Stanford University Press.

Flache, A., Mäs, M., Feliciani, T., Chattoe-Brown, E., Deffuant, G., Huet, S., \& Lorenz, J. (2017). Models of Social Influence: Towards the Next Frontiers. Journal of Artificial Societies \& Social Simulation, 20(4).

Grossmann, I., \& Varnum, M. E. (2015). Social structure, infectious diseases, disasters, secularism, and cultural change in America. Psychological Science, 26(3), 311-324. 
Gupta, A. K., \& Nadarajah, S. (2004). Handbook of beta distribution and its applications. Boca Raton, FL: CRC Press.

Haider, M., \& Kreps, G. L. (2004). Forty years of diffusion of innovations: utility and value in public health. Journal of health communication, 9(S1), 3-11.

Harton, H. C., \& Bourgeois, M. J. (2003). Cultural elements emerge from dynamic social impact. In M. Schaller \& C. S. Crandall (Eds.), The psychological foundations of culture (pp. 4175). New York, NY: Psychology Press.

Harton, H. C., \& Bullock, M. (2007). Dynamic social impact: A theory of the origins and evolution of culture. Social and Personality Psychology Compass, 1(1), 521-540.

Hastie, R., \& Kameda, T. (2005). The Robust Beauty of Majority Rules in Group Decisions. Psychological Review, 112(2), 494-508.

Heine, S. J., Foster, J. A. B., \& Spina, R. (2009). Do birds of a feather universally flock together? Cultural variation in the similarity-attraction effect. Asian Journal of Social Psychology, 12(4), 247-258.

Henrich, J. (2001). Cultural transmission and the diffusion of innovations: Adoption dynamics indicate that biased cultural transmission is the predominate force in behavioral change. American Anthropologist, 103(4), 992-1013.

Henrich, J. (2004). Cultural group selection, coevolutionary processes and large-scale cooperation. Journal of Economic Behavior \& Organization, 53(1), 3-35.

Henrich, J., \& Broesch, J. (2011). On the nature of cultural transmission networks: evidence from Fijian villages for adaptive learning biases. Philosophical Transactions of the Royal Society B: Biological Sciences, 366(1567), 1139-1148. 
Hofstede, G., \& McCrae, R. R. (2004). Personality and culture revisited: Linking traits and dimensions of culture. Cross-cultural research, 38(1), 52-88.

Hoshino-Browne, E. (2012). Cultural Variations in Motivation for Cognitive Consistency: Influences of Self-Systems on Cognitive Dissonance. Social and Personality Psychology Compass, 6(2), 126-141.

Jackson, J. C., Rand, D., Lewis, K., Norton, M. I., \& Gray, K. (2017). Agent-based modeling: A guide for social psychologists. Social Psychological and Personality Science, 8(4), 387395.

Jackson, M. O. (2010). Social and economic networks. Princeton, NJ: Princeton University Press.

Kameda, T., Takezawa, M., \& Hastie, R. (2003). The logic of social sharing: An evolutionary game analysis of adaptive norm development. Personality and Social Psychology Review, $7(1), 2-19$.

Kao, A. B., \& Couzin, I. D. (2014). Decision accuracy in complex environments is often maximized by small group sizes. Proceedings of the Royal Society B: Biological Sciences, 281(1784), 20133305.

Kashima, Y., Wilson, S., Lusher, D., Pearson, L. J., \& Pearson, C. (2013). The acquisition of perceived descriptive norms as social category learning in social networks. Social Networks, 35(4), 711-719.

Kempe, M., \& Mesoudi, A. (2014). An experimental demonstration of the effect of group size on cultural accumulation. Evolution and Human Behavior, 35(4), 285-290.

Kenney, P. J., \& Rice, T. W. (1994). The psychology of political momentum. Political Research Quarterly, 47(4), 923-938. 
Kerr, N. L., \& Tindale, R. S. (2004). Group performance and decision making. Annual review of psychology, 55, 623-655.

Kim, H., \& Markus, H. R. (1999). Deviance or uniqueness, harmony or conformity? A cultural analysis. Journal of Personality and Social Psychology, 77(4), 785-800.

Kojadinovic, I., \& Yan, J. (2010). Modeling multivariate distributions with continuous margins using the copula R package. Journal of Statistical Software, 34(9), 1-20.

Kruglanski, A. W., Webster, D. M., \& Klem, A. (1993). Motivated resistance and openness to persuasion in the presence or absence of prior information. Journal of Personality and Social Psychology, 65(5), 861-876.

Laar, C. V., Levin, S., Sinclair, S., \& Sidanius, J. (2005). The effect of university roommate contact on ethnic attitudes and behavior. Journal of Experimental Social Psychology, 41(4), 329-345.

Latané, B. (1996). Dynamic social impact: The creation of culture by communication. Journal of Communication, 46(4), 13-25.

Latané, B., Liu, J. H., Nowak, A., Bonevento, M., \& Zheng, L. (1995). Distance matters: Physical space and social impact. Personality and Social Psychology Bulletin, 21(8), 795805.

Liu, P., Chan, D., Qiu, L., Tov, W., \& Tong, V. J. C. (2018). Effects of Cultural TightnessLooseness and Social Network Density on Expression of Positive and Negative Emotions: A Large-Scale Study of Impression Management by Facebook Users. Personality and Social Psychology Bulletin, 0146167218770999.

MacCoun, R. J. (2012). The burden of social proof: Shared thresholds and social influence. Psychological Review, 119(2), 345. 
Mason, W. A., Conrey, F. R., \& Smith, E. R. (2007). Situating social influence processes: Dynamic, multidirectional flows of influence within social networks. Personality and Social Psychology Review, 11(3), 279-300.

Moscovici, S. (1980). Toward a theory of conversion behavior. Advances in experimental social psychology, 13, 209-239.

Muthukrishna, M., \& Henrich, J. (2016). Innovation in the collective brain. Philosophical Transactions of the Royal Society of London B: Biological Sciences, 371(1690).

Muthukrishna, M., Morgan, T. J. H., \& Henrich, J. (2016). The When and Who of Social Learning and Conformist Transmission. Evolution and Human Behavior, 37(1), 10-20.

Muthukrishna, M., Shulman, B. W., Vasilescu, V., \& Henrich, J. (2013). Sociality influences cultural complexity. Proceedings of the Royal Society B: Biological Sciences, 281(1774), 20132511.

Nadeau, R., Cloutier, E., \& Guay, J.-H. (1993). New evidence about the existence of a bandwagon effect in the opinion formation process. International Political Science Review, 14(2), 203-213.

Nemeth, C. J. (1986). Differential contributions of majority and minority influence. Psychological Review, 93(1), 23.

Ng, K. Y., \& Van Dyne, L. (2001). Individualism-collectivism as a boundary condition for effectiveness of minority influence in decision making. Organizational Behavior and Human Decision Processes, 84(2), 198-225.

Nowak, A. (2004). Dynamical minimalism: Why less is more in psychology. Personality and Social Psychology Review, 8(2), 183-192. 
Nowak, A., Szamrej, J., \& Latané, B. (1990). From private attitude to public opinion: A dynamic theory of social impact. Psychological Review, 97(3), 362-376.

Orji, R. (2016). Persuasion and Culture: Individualism-Collectivism and Susceptibility to Influence Strategies. Paper presented at the PPT@ PERSUASIVE.

Pfau, J., Kirley, M., \& Kashima, Y. (2013). The co-evolution of cultures, social network communities, and agent locations in an extension of Axelrod's model of cultural dissemination. Physica A: Statistical Mechanics and its Applications, 392(2), 381-391.

Pollet, T. V., Roberts, S. G. B., \& Dunbar, R. I. M. (2011). Extraverts have larger social network layers: But do not feel emotionally closer to individuals at any layer. Journal of Individual Differences, 32(3), 161-169.

Rogers, E. M. (2003). Diffusion of innovations (5th ed.). New York, NY: Free Press.

Schaller, M., Conway, L. G., III, \& Tanchuk, T. L. (2002). Selective pressures on the once and future contents of ethnic stereotypes: effects of the communicability of traits. Journal of Personality and Social Psychology, 82(6), 861.

Schug, J., Yuki, M., Horikawa, H., \& Takemura, K. (2009). Similarity attraction and actually selecting similar others: How cross-societal differences in relational mobility affect interpersonal similarity in Japan and the USA. Asian Journal of Social Psychology, 12(2), 95-103.

Singelis, T. M., Triandis, H. C., Bhawuk, D. P., \& Gelfand, M. J. (1995). Horizontal and vertical dimensions of individualism and collectivism: A theoretical and measurement refinement. Cross-cultural research, 29(3), 240-275.

Smaldino, P. E. (2017). Models are stupid, and we need more of them. Computational social psychology, 311-331. 
Spencer-Rodgers, J., Williams, M. J., \& Peng, K. (2010). Cultural differences in expectations of change and tolerance for contradiction: A decade of empirical research. Personality and Social Psychology Review, 14(3), 296-312.

Tesser, A. (1993). The importance of heritability in psychological research: the case of attitudes. Psychological Review, 100(1), 129-142.

Ugander, J., Karrer, B., Backstrom, L., \& Marlow, C. (2011). The anatomy of the facebook social graph. arXiv preprint arXiv:1111.4503.

Valente, T. W. (1995). Network models of the diffusion of innovations (Vol. 2). Cresskill, NJ: Hampton Press.

Vallacher, R. R., Read, S. J., \& Nowak, A. (2002). The dynamical perspective in personality and social psychology. Personality and Social Psychology Review, 6(4), 264-273.

Varnum, M. E., \& Grossmann, I. (2017). Cultural change: The how and the why. Perspectives on Psychological Science, 12(6), 956-972.

Weisbuch, G., Deffuant, G., \& Amblard, F. (2005). Persuasion dynamics. Physica A: Statistical Mechanics and its Applications, 353, 555-575.

Wejnert, B. (2002). Integrating models of diffusion of innovations: A conceptual framework. Annual Review of Sociology, 28, 297-326.

Wilken, B., Miyamoto, Y., \& Uchida, Y. (2011). Cultural influences on preference consistency: Consistency at the individual and collective levels. Journal of Consumer Psychology, $21(3), 346-353$.

Wood, W., Lundgren, S., Ouellette, J. A., Busceme, S., \& Blackstone, T. (1994). Minority influence: A meta-analytic review of social influence processes. Psychological Bulletin, $115(3), 323-345$. 
Zou, X., Tam, K.-P., Morris, M. W., Lee, S.-1., Lau, I. Y.-M., \& Chiu, C.-y. (2009). Culture as common sense: Perceived consensus versus personal beliefs as mechanisms of cultural influence. Journal of Personality and Social Psychology, 97(4), 579-597. 
Table 1

Structural properties of social networks that emerged in Phase 2 of the simulations, as a function of mean level of extraversion within the population. (Tabled values are means computed across 100 simulations for each of the three levels of extraversion; standard deviations around these means are in parentheses).

\begin{tabular}{|c|c|c|c|}
\hline $\begin{array}{c}\text { Population-wide Mean } \\
\text { Level of Extraversion }\end{array}$ & $\begin{array}{c}\text { Characteristic Path } \\
\text { Length }\end{array}$ & $\begin{array}{l}\text { Clustering } \\
\text { Coefficient }\end{array}$ & Segree Distribution \\
\hline Low & $3.82(.04)$ & $.13(.005)$ & $.56(.07)$ \\
\hline Medium & $3.49(.02)$ & $.15(.004)$ & $.37(.07)$ \\
\hline High & $3.23(.02)$ & $.16(.003)$ & $.25(.08)$ \\
\hline
\end{tabular}


Table 2

Number of influence opportunities required for a 2/3 super-majority to emerge, as a function of population-wide mean values of influenceability (representing low-, moderate-, and high-

influenceability cultures). Tabled values are means computed across 1200 simulations for each of the three levels of influenceability; standard deviations around these means are in parentheses. (Values are provided separately for primarily simulations and for additional supplemental simulations; see text for details on different sets of simulations).

\begin{tabular}{|c|c|c|c|}
\hline \multicolumn{4}{|c|}{ Primary simulations } \\
\hline & \multicolumn{3}{|c|}{$\begin{array}{l}\text { Population-Wide Mean Level of } \\
\text { Influenceability }\end{array}$} \\
\hline & Low & Moderate & High \\
\hline Influence Opportunities Required for & 174.31 & 130.07 & 110.74 \\
\hline Super-Majority to Emerge & $(143.19)$ & $(103.81)$ & $(94.83)$ \\
\hline \multicolumn{4}{|c|}{$\begin{array}{l}\text { Supplemental simulations } \\
\text { (representing a "conformist transmission" bias in which majority opinion is overweighted) }\end{array}$} \\
\hline & \multicolumn{3}{|c|}{$\begin{array}{l}\text { Population-Wide Mean Level of } \\
\text { Influenceability }\end{array}$} \\
\hline & Low & Moderate & High \\
\hline Influence Opportunities Required for & 36.51 & 27.29 & 23.60 \\
\hline Super-Majority to Emerge & $(55.26)$ & $(42.10)$ & $(35.16)$ \\
\hline
\end{tabular}


Table 3

Likelihood that the initially unpopular belief_initially held by a single "lone ideologue"successfully spread to $50 \%$ of the entire population, as a function of population-wide mean values of influenceability and extraversion. (Tabled values are provided separately for simulations in which individuals' influenceability and extraversion values were either independent or non-independent; see text for details on different sets of simulations.)

\begin{tabular}{|c|c|c|c|}
\hline \multicolumn{4}{|c|}{ Individuals' Conformity and Extraversion Values are Independent: } \\
\hline \multirow{2}{*}{$\begin{array}{c}\text { Population-wide Mean Level of } \\
\text { Extraversion } \\
\end{array}$} & \multicolumn{3}{|c|}{$\begin{array}{c}\text { Population-wide Mean Level of } \\
\text { Influenceability }\end{array}$} \\
\hline & Low & Moderate & High \\
\hline Low & $24.4 \%$ & $36.6 \%$ & $50.9 \%$ \\
\hline Moderate & $21.5 \%$ & $35.2 \%$ & $46.1 \%$ \\
\hline High & $17.5 \%$ & $32.7 \%$ & $38.5 \%$ \\
\hline \multicolumn{4}{|c|}{ Individuals' Conformity and Extraversion Values are Non-independent $(r=-.3)$} \\
\hline \multirow{2}{*}{$\begin{array}{c}\text { Population-wide Mean Level of } \\
\text { Extraversion }\end{array}$} & \multicolumn{3}{|c|}{$\begin{array}{c}\text { Population-wide Mean Level of } \\
\text { Influenceability }\end{array}$} \\
\hline & Low & Moderate & High \\
\hline Low & $30.9 \%$ & $43.0 \%$ & $54.1 \%$ \\
\hline Moderate & $26.7 \%$ & $36.0 \%$ & $46.3 \%$ \\
\hline High & $19.2 \%$ & $29.9 \%$ & $40.2 \%$ \\
\hline
\end{tabular}


Table 4

Likelihood that the initially unpopular belief-initially held by a single adherent with a non-zero influenceability value-successfully spread to $50 \%$ of the entire population, as a function of population-wide mean values of influenceability and extraversion. (Tabled values are provided separately for simulations in which the primary adherent had an influenceability value of .01 or .10; see text for details on different sets of simulations.).

\begin{tabular}{|c|c|c|c|}
\hline \multicolumn{4}{|c|}{ Primary adherent has an influenceability value of .01 } \\
\hline \multirow{2}{*}{$\begin{array}{l}\text { Population-wide Mean Level of } \\
\text { Extraversion } \\
\end{array}$} & \multicolumn{3}{|c|}{$\begin{array}{l}\text { Population-wide Mean Level of } \\
\text { Conformity }\end{array}$} \\
\hline & Low & Moderate & High \\
\hline Low & $3.1 \%$ & $7.5 \%$ & $8.9 \%$ \\
\hline Moderate & $2.6 \%$ & $5.3 \%$ & $9.1 \%$ \\
\hline High & $2.3 \%$ & $5.3 \%$ & $7.3 \%$ \\
\hline \multicolumn{4}{|c|}{ Primary adherent has an influenceability value of .10 } \\
\hline \multirow{2}{*}{$\begin{array}{l}\text { Population-wide Mean Level of } \\
\text { Extraversion }\end{array}$} & \multicolumn{3}{|c|}{$\begin{array}{l}\text { Population-wide Mean Level of } \\
\text { Conformity }\end{array}$} \\
\hline & Low & Moderate & High \\
\hline Low & $0.4 \%$ & $0.3 \%$ & $1.0 \%$ \\
\hline Moderate & $0.1 \%$ & $0.6 \%$ & $0.9 \%$ \\
\hline High & $0.1 \%$ & $0.4 \%$ & $0.5 \%$ \\
\hline
\end{tabular}


Figure 1. Results depicting main effects that population-wide mean levels of influenceability and extraversion had on diffusion of an initially unpopular belief. These results depict the percent of simulations in which the initially unpopular belief_-initially held by just a single "lone ideologue"- successfully spread to $50 \%$ of the entire population. Results reveal that initially unpopular beliefs spread more readily within simulated cultures characterized by higher levels of susceptibility to social influence and by fewer acquaintances within individuals' personal social networks (i.e., a less dense social network structure within the population).
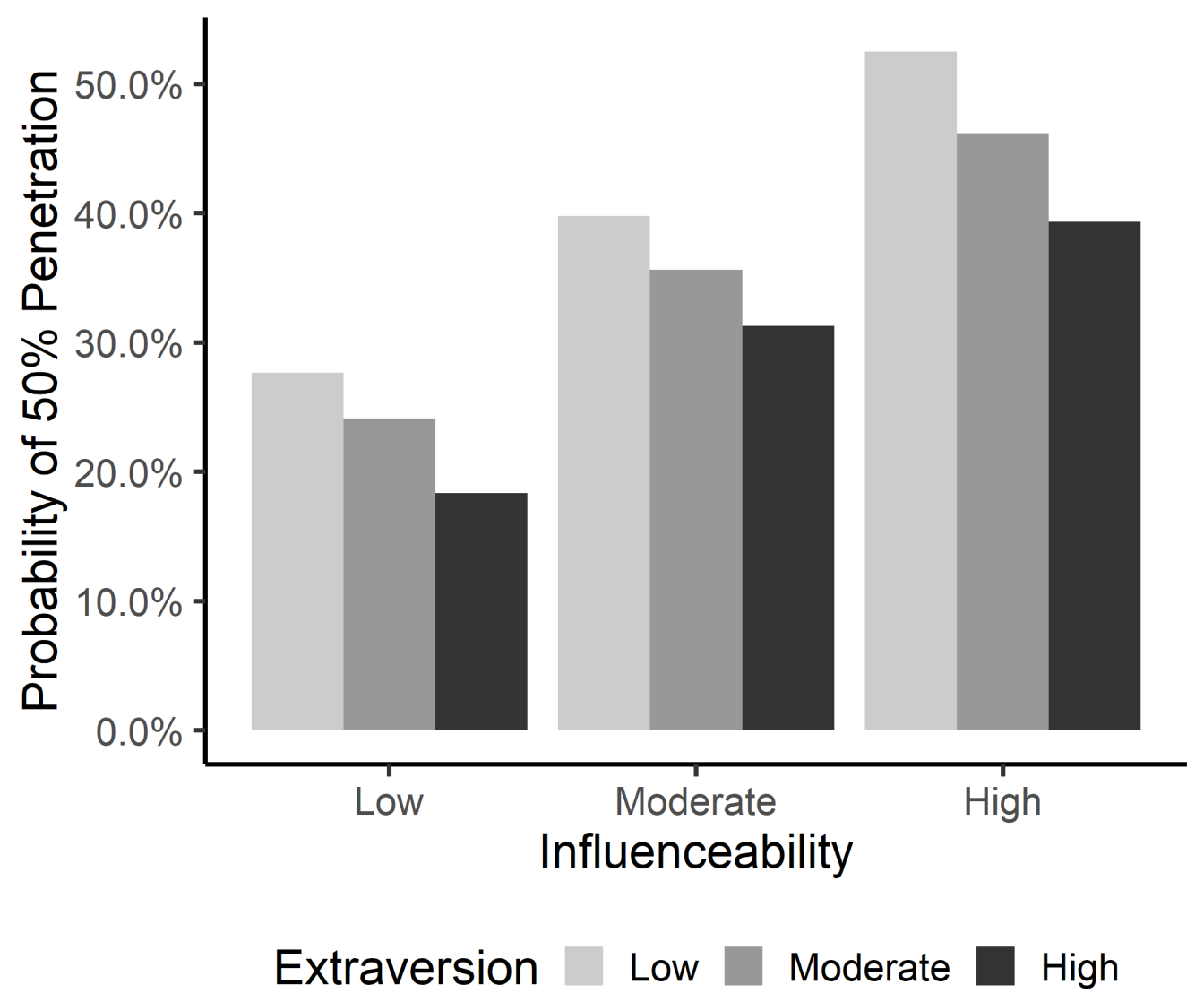